\title{
Stability of towed wheels in cornering manoeuvre
}

\author{
Henrik Sykora \\ Department of Applied Mechanics, Budapest University of Technology and Economics, Budapest, Hungary
}

Dénes Takács

MTA-BME Research Group on Dynamics of Machines and Vehicles, Budapest, Hungary

\begin{abstract}
A simple mechanical model of a towed rigid wheel is constructed in which the elasticity of the king pin is considered in both the longitudinal and the lateral directions. The equations of motion are derived and presented for the rolling case. The cornering manoeuvre of the towed wheel is investigated with respect to the linear stability and the non-linear vibrations. Linear stability boundary of the uniform motion is determined for different cornering radii. The sense of the related Hopf bifurcation is determined by numerical continuation technique. Bifurcation diagrams are composed to show the basin of attraction of the stable uniform motion.
\end{abstract}

\section{INTRODUCNTION}

The shimmy motion of the towed wheel is a well-known phenomenon of everyday life. The swivelling wheels of shopping trolleys and baby strollers often show this motion that strongly reduces the manoeuvrability of these vehicles. But shimmy is also an unwanted vibration of bicycles, motorcycles (Sharp et al., 2004; Cossalter, 2006) and air-plane gears (Besselink, 2000; Terkovics et al., 2012). The simplest mechanical models of these vehicle systems, which can be efficiently used to capture shimmy, are very similar to each other. Namely, the different combinations of rigid/elastic wheel and rigid/elastic suspension in low degree-of-freedom mechanical models are commonly used to analyse shimmy, and there are both analytical and numerical results that can help to eliminate shimmy (see, for example, Pacejka (2002); Takács et al. (2008)). The majority of these analyses focuses on the vibration around the rectilinear motion of the wheel.

It can be observed in practice that shimmy can be induced easier during cornering. On the one hand, large perturbations of the towed wheel can emerge at the beginning stage of the cornering when the cornering radius is suddenly changed. These large perturbation can lead to unwanted self-excited vibration even in linearly stable parameter domain if an unstable limitcyle is also present. On the other hand, the cornering is generally a more disadvantageous manoeuvre relative to the rectilinear motion due to the enhanced lateral force at the wheel/ground contact.

In this study, a low degree-of-freedom mechanical model is constructed based on Takács et al. (2008). Here, the longitudinal elasticity of the suspension is also considered, which provides an extra degree of freedom for the system. The linear stability of the uniform circular motion is analysed and stability charts are presented for the different values of the cornering radius. Numerical continuation technique is applied to determine the sense of the Hopf bifurcation at the linear stability boundary.

\section{MECHANICAL MODEL}

The mechanical model is shown in Figure 1. A rigid wheel of radius $R$ is attached to a rigid caster of length $l$. The distance between the centre of gravity $\mathrm{C}$ of the caster and the king pin A is characterized by $l_{\mathrm{c}}$. The mass of the caster and the mass moment of inertia of the caster about the $z$ axis at $\mathrm{C}$ are denoted by $m_{\mathrm{c}}$ and $J_{\mathrm{c} z}$, respectively. Similar notations with the subscript $\mathrm{w}$ are introduced for the mass and mass moments of inertia parameters of the wheel. 


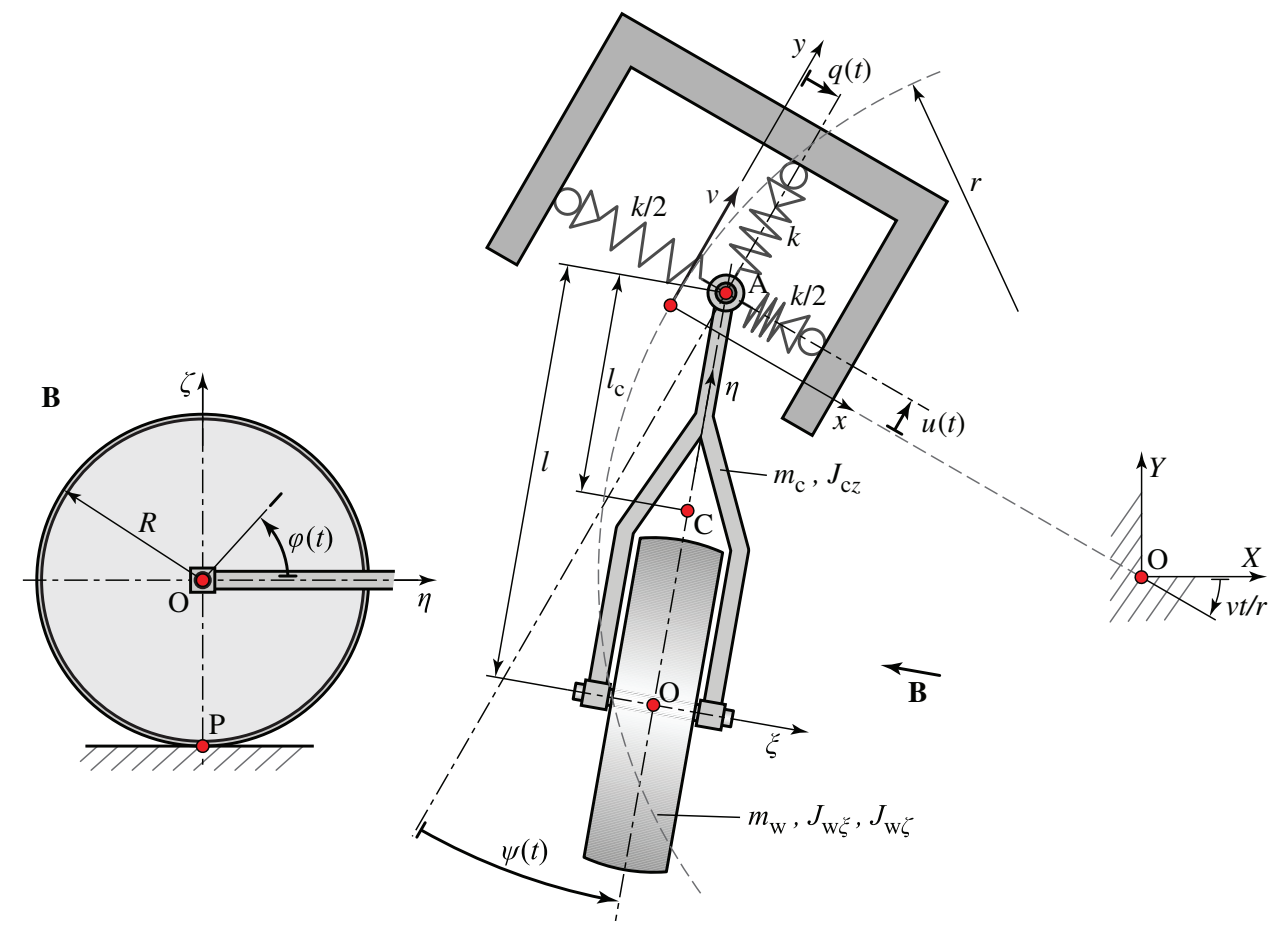

Figure 1. The mechanical model

The king pin A is driven by an elastic suspension system but it is assumed that the king pin axis is kept normal to the ground by the suspension. Elasticities are only considered in the lateral and in the longitudinal directions with the uniform spring stiffness $k$. The suspension system is towed by constant speed $v$ on a circular orbit with radius $r$. The lateral and the longitudinal displacements of the king pin are described by $q(t)$ and $u(t)$, respectively. The caster angle is denoted by $\psi(t)$ while the revolution of the wheel around its axis of rotation is defined by $\varphi(t)$.

The pure rolling of the wheel is considered, i.e. the wheel contact point $\mathrm{P}$ has zero velocity relative to the ground $\left(v_{P}=0\right)$. This kinematic constraint of rolling can be formulated as

$$
\begin{aligned}
\dot{q}+l \dot{\psi} \cos \psi-R \dot{\varphi} \sin \psi+\frac{v}{r}(u-l \cos \psi) & =0, \\
\dot{u}+l \dot{\psi} \sin \psi+R \dot{\varphi} \cos \psi-\frac{v}{r}(q+l \sin \psi)+v & =0 .
\end{aligned}
$$

The equations of motion of the so-formed non-holonomic system can be efficiently derived by using the Lagrange-equation of the second kind with additional Lagrange multipliers for the constraining forces that relates to the kinematic constraints. Another possibility, which is applied here, is the use of the Appell-Gibbs equations, which requires the introduction of properly chosen pseudo velocities. In our case, two pseudo velocities $\beta_{1}$ and $\beta_{2}$ have to be chosen since the difference of the number of the generalized coordinates and the number of the kinematic constraining equations is two. An appropriate choice for the pseudo velocities can be:

$$
\beta_{1}=\dot{\psi} \quad \text { and } \quad \beta_{2}=\dot{u} .
$$

By means of the definitions in Eqn. (2) together with the kinematic constraining equations in Eqn. (1), one can express the generalized velocities as the functions of the generalized coordinates and the pseudo velocities:

$$
\begin{aligned}
& \dot{q}=\frac{1}{\cos \psi}\left(\left(\frac{v}{r}-\beta_{1}\right) l-\left(v+\frac{v}{r} q+\beta_{2}\right) \sin \psi\right)-\frac{v}{r} u, \\
& \dot{u}=\beta_{2}, \\
& \dot{\psi}=\beta_{1}, \\
& \dot{\varphi}=-\frac{1}{R \cos \psi}\left(v-\frac{v}{r} q+\beta_{2}-\left(\frac{v}{r}-\beta_{1}\right) l \sin \psi\right) .
\end{aligned}
$$

The Appell-Gibbs method gives the governing equation of the system in the form of a system of first order ordinary differential equations (ODEs), where state space coordinates are $\beta_{1}, \beta_{2}, q, u$, $\psi$ and $\varphi$. Due to the fact that the derivation of the equation of motion provides extensive formulas for our mechanical model, we only present the main steps of the derivation process. 
The Appell-Gibbs equations can be formulated as

$$
\frac{\partial \mathcal{A}}{\partial \dot{\beta}_{r}}=\Gamma_{r},
$$

where $r=1,2$. The so-called energy of acceleration $\mathcal{A}$ can be calculated as

$$
\begin{aligned}
& \mathcal{A}=\frac{1}{2} m_{\mathrm{c}} \mathbf{a}_{\mathrm{C}} \cdot \mathbf{a}_{\mathrm{C}}+\frac{1}{2} \boldsymbol{\varepsilon}_{\mathrm{c}}^{\mathrm{T}} \mathbf{J}_{\mathrm{c}} \boldsymbol{\varepsilon}_{\mathrm{c}}+\boldsymbol{\varepsilon}_{\mathrm{c}}^{\mathrm{T}}\left(\boldsymbol{\omega}_{\mathrm{c}} \times\left(\mathbf{J}_{\mathrm{c}} \boldsymbol{\omega}_{\mathrm{c}}\right)\right) \\
& +\frac{1}{2} m_{\mathrm{w}} \mathbf{a}_{\mathrm{O}} \cdot \mathbf{a}_{\mathrm{O}}+\frac{1}{2} \varepsilon_{\mathrm{w}}^{\mathrm{T}} \mathbf{J}_{\mathrm{w}} \boldsymbol{\varepsilon}_{\mathrm{w}}+\boldsymbol{\varepsilon}_{\mathrm{w}}^{\mathrm{T}}\left(\boldsymbol{\omega}_{\mathrm{w}} \times\left(\mathbf{J}_{\mathrm{w}} \boldsymbol{\omega}_{\mathrm{w}}\right)\right)+\ldots .
\end{aligned}
$$

Here, $\mathbf{a}_{\mathrm{C}}$ and $\mathbf{a}_{\mathrm{O}}$ are the accelerations of the centres of gravity of the caster and wheel, respectively. The notations $\varepsilon_{\mathrm{c}}$ and $\varepsilon_{\mathrm{w}}$ refer to the angular accelerations while $\boldsymbol{\omega}_{\mathrm{c}}$ and $\boldsymbol{\omega}_{\mathrm{w}}$ refer to the angular velocities of the caster and the wheel, respectively. The mass moment of inertia matrices of the caster and the wheel are denoted by $\mathbf{J}_{\mathrm{c}}$ and $\mathbf{J}_{\mathrm{w}}$, respectively. Based on Eqn. (3), the energy of acceleration can be calculated as the function of the pseudo accelerations $\left(\dot{\beta}_{1}\right.$ and $\left.\dot{\beta}_{2}\right)$, pseudo velocities $\left(\beta_{1}\right.$ and $\left.\beta_{2}\right)$ and the general coordinates $(q, u, \psi$ and $\varphi)$.

The pseudo forces $\Gamma_{1}$ and $\Gamma_{2}$ can be calculated via the virtual power of the active forces. In our case, the spring forces at the king ping have only non-zero virtual power, namely:

$$
\delta P=\mathbf{F}_{\mathrm{A}}^{\mathrm{T}} \delta \mathbf{v}_{\mathrm{A}} \equiv \Gamma_{1} \delta \beta_{1}+\Gamma_{2} \delta \beta_{2},
$$

where $\delta$ denotes virtual quantities, $\mathbf{F}_{\mathrm{A}}$ is the force that acts on the king pin and $\mathbf{v}_{\mathrm{A}}$ is the velocity of the king pin. The pseudo forces can be calculated as

$$
\begin{aligned}
& \Gamma_{1}=k l q / \cos \psi, \\
& \Gamma_{2}=k(q \tan \psi-u) .
\end{aligned}
$$

Based on Eqn. (4), the following equations can be obtained after some algebraic manipulation:

$$
\begin{aligned}
& \left(J_{\mathrm{c} z}+J_{\mathrm{w} \zeta}+m_{\mathrm{c}}\left(l-l_{\mathrm{c}}\right)^{2}\right) \dot{\beta}_{1} \\
& +\frac{m_{\mathrm{c}}\left(l-l_{\mathrm{c}}\right)}{\cos \psi}\left(\beta_{1}^{2} l \sin \psi-\left(v+\frac{v}{r}(q+2 l \sin \psi)-\beta_{2}\right) \beta_{1}\right) \\
& -\frac{v}{r} \frac{m_{\mathrm{c}}\left(l-l_{\mathrm{c}}\right)}{\cos \psi}\left(v-\frac{v}{r}(q-l \sin \psi)+\beta_{2}\right)-k l(q \cos \psi+u \sin \psi)=0, \\
& -\frac{J_{\mathrm{w} \xi}+\left(m_{\mathrm{c}}+m_{\mathrm{w}}\right) R^{2}}{R^{2} \cos ^{2} \psi}\left(\dot{\beta}_{1} l \sin \psi+\dot{\beta}_{2}\right)+\left(\frac{m_{\mathrm{c}}\left(l-l_{\mathrm{c}}\right)}{\cos \psi}-\frac{J_{\mathrm{w} \xi}+\left(m_{\mathrm{w}}+m_{\mathrm{c}}\right) R^{2}}{R^{2} \cos ^{3} \psi} l\right) \beta_{1}{ }^{2} \\
& +\left(\frac{v}{r}\left(\frac{2 m_{\mathrm{c}}\left(l-l_{\mathrm{c}}\right)}{\cos \psi}-\frac{J_{\mathrm{w} \xi}+\left(m_{\mathrm{w}}+m_{\mathrm{c}}\right) R^{2}}{R^{2} \cos ^{3} \psi} \sin \psi\right)+\frac{J_{\mathrm{w} \xi}+\left(m_{\mathrm{w}}+m_{\mathrm{c}}\right) R^{2}}{r R^{2} \cos ^{3} \psi}\left(v q-r \beta_{2}\right) \sin \psi\right) \beta_{1} \\
& -\frac{v}{r} \frac{J_{\mathrm{w} \xi}+\left(m_{\mathrm{c}}+m_{\mathrm{w}}\right) R^{2}}{R^{2} \cos ^{2} \psi} \beta_{2} \tan \psi+\frac{v^{2}}{r^{2}} \frac{J_{\mathrm{w} \xi}+\left(m_{\mathrm{w}}+m_{\mathrm{c}}\right) R^{2}}{R^{2} \cos ^{2} \psi}(q \tan \psi-u)+k(q \tan \psi-u) \\
& +\frac{v^{2}}{r^{2}}\left(\frac{m_{\mathrm{c}}\left(3 l-l_{\mathrm{c}}\right)+2 m_{\mathrm{w}} l+m_{\mathrm{c}}\left(l-l_{\mathrm{c}}\right) \cos 2 \psi}{2 \cos ^{3} \psi}+\frac{J_{\mathrm{w} \xi} l}{R^{2} \cos ^{3} \psi}-\frac{J_{\mathrm{w} \xi}+\left(m_{\mathrm{w}}+m_{\mathrm{c}}\right) R^{2}}{R^{2} \cos ^{3} \psi} r \sin \psi\right)=0 .
\end{aligned}
$$

These coupled to Eqn. (3) describe the motion of our mechanical model uniquely. Since the wheel rotation angle $\varphi$ does not appear in the equations, which means that $\varphi$ is a cyclic coordinate, $\varphi$ can be eliminated. Thus, the system can be investigated in a five dimensional state space.

\section{LINEAR STABILITY ANALYSIS}

The equations of motion Eqns. (3) and (8) can be rearranged as $\dot{\mathbf{x}}=\mathbf{f}(\mathbf{x})$, and the fix points of the system can be calculated by means of $\mathbf{f}\left(\mathbf{x}_{0}\right)=\mathbf{0}$. In engineering point of view, we can say that we look for the uniform motion of the towed cornering wheel, which motion is characterized by $q(t) \equiv q_{0}, u(t) \equiv u_{0}$ and $\psi(t) \equiv \psi_{0}$ while the wheel rotation angle can be given as $\varphi(t)=$ $\omega_{0} t$. After the substitution of these into the governing equations (Eqns. (3) and (8)), we obtain transcendent algebraic equations with respect to $q_{0}, u_{0}, \psi_{0}$ and $\omega_{0}$. Thus, the uniform motion can be determined numerically only.

Let us consider the parameters of a realistic swivelling wheel: $m_{\mathrm{w}}=0.3519 \mathrm{~kg}, m_{\mathrm{c}}=$ $0.0668 \mathrm{~kg}, J_{\mathrm{w} \xi}=4.63 \times 10^{-5} \mathrm{kgm}^{2}, J_{\mathrm{w} \zeta}=2.38 \times 10^{-5} \mathrm{kgm}^{2}, J_{\mathrm{c} z}=3.48 \times 10^{-6} \mathrm{kgm}^{2}$, $R=0.04 \mathrm{~m}, l_{\mathrm{c}}=0.012 \mathrm{~m}$ and $k=100 \mathrm{~N} / \mathrm{m}$.

In Figure 2, the coordinates characterizing the uniform motion are plotted against the towing speed $v$ for the caster length $l=0.08 \mathrm{~m}$ and turning radius $r=1 \mathrm{~m}$. As it can be seen the longitudinal displacement $u_{0}$ of the king pin is practically zero, which suggests that this degree of freedom of our mechanical model can be neglected. On the contrary, it can be shown that the longitudinal degree of freedom has qualitative effect on the linear stability of the towed wheel. 

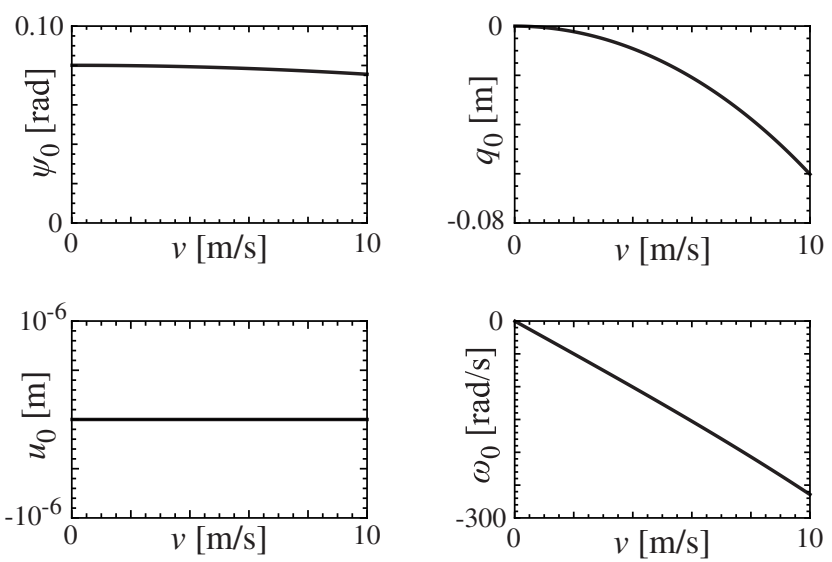

Figure 2. The variation of the uniform motion with respect to the towing speed for $l=0.08 \mathrm{~m}$ and $r=1 \mathrm{~m}$.

In order to investigate the linear stability of the uniform motion, the equations of motion are linearised around this motion, and the eigenvalues of the Jacobian are calculated. A stability chart is shown in Figure 3. The stability boundaries are constructed in the plane of the towing speed $v$ and caster length $l$ for different cornering radii $r$. The stability chart is shaded for $r=1 \mathrm{~m}$, the shaded domain is linearly stable. In case of the rectilinear motion $(r \rightarrow \infty)$, there exist a critical caster length over it the uniform motion is stable for any speed (see in Takács et al. (2008)). In case of cornering, there always exists a critical speed over it the uniform motion is linearly unstable independently of the caster length. The smaller the turning radius, the smaller this critical speed.

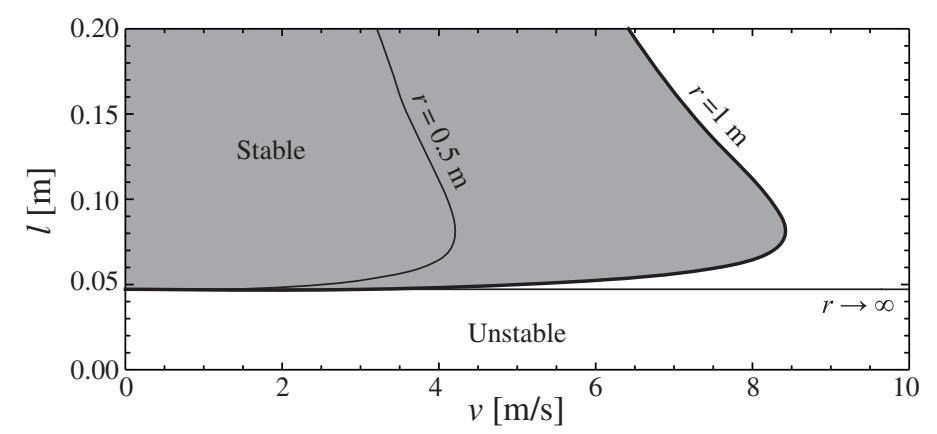

Figure 3. Linear stability chart in the plane of the towing speed $v$ and caster length $l$ for different cornering radii $r$

In our study, we consider the pure rolling of the wheel without any restriction to the contact force at the contact point P. In order to check that the determined uniform motion can exist in practice, the contact force that emerge in case of the uniform motion is calculated. The friction force $F_{\mathrm{f}}$ and the normal force $N$ are

$$
\begin{aligned}
& F_{\mathrm{f}}=\frac{v^{2}}{r^{2} \cos \psi_{0}} \sqrt{\left(\frac{J_{\mathrm{w} \xi}^{2}}{R^{4}}\left(u_{0}+\left(r-q_{0}\right) \tan \psi_{0}-\frac{l}{\cos \psi_{0}}\right)^{2}+\frac{\left(m_{\mathrm{w}} l+m_{\mathrm{c}} l_{\mathrm{c}}\right)^{2}}{l^{2}}\left(q_{0}-r+l \sin \psi_{0}\right)^{2}\right)}, \\
& N=-F_{\mathrm{A} z}+\left(m_{\mathrm{w}}+m_{\mathrm{c}}\right) g,
\end{aligned}
$$

respectively, where $F_{\mathrm{A} z}$ is the vertical force that acts on the king pin due to the vertical load of the towed wheel. The acceleration of gravity is $g=9.81 \mathrm{~m} / \mathrm{s}^{2}$. Using these formulas we marked the boundary of the rolling $\left(\mathbf{v}_{\mathrm{P}}=\mathbf{0}\right.$ and $\left.F_{\mathrm{f}}<\mu_{\mathrm{S}} N\right)$ and the sliding cases in Figure 4 for the static coefficient of friction $\mu_{\mathrm{s}}=1$ and for different values of the vertical force. The curves are constructed for the turning radius $r=1 \mathrm{~m}$. As it can be observed, the calculated uniform motion can exist for realistic vertical loads of the wheel. 


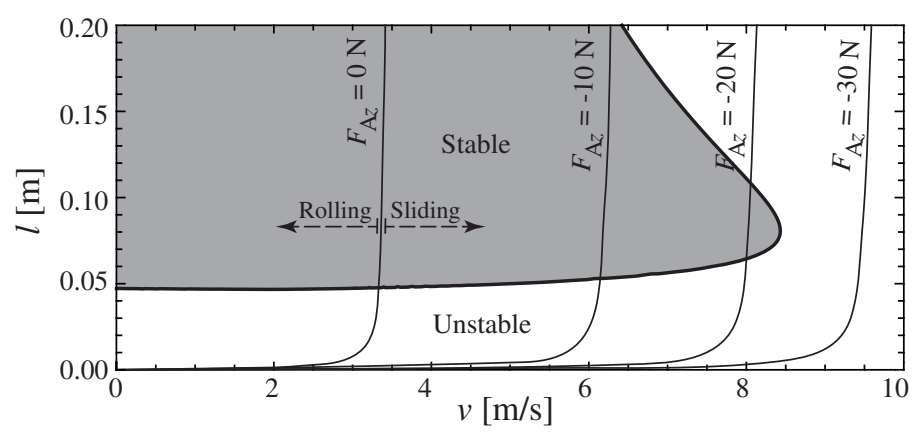

Figure 4. The boundary between the rolling and the sliding domain illustrated in the linear stability chart for different vertical loads

\section{NUMERICAL BIFURCATION ANALYSIS}

The governing equation of the system are implemented in the numerical bifurcation software AUTO (Doedel et al., 1997) and the behaviour of the towed wheel is investigated in the non-linear domain too.

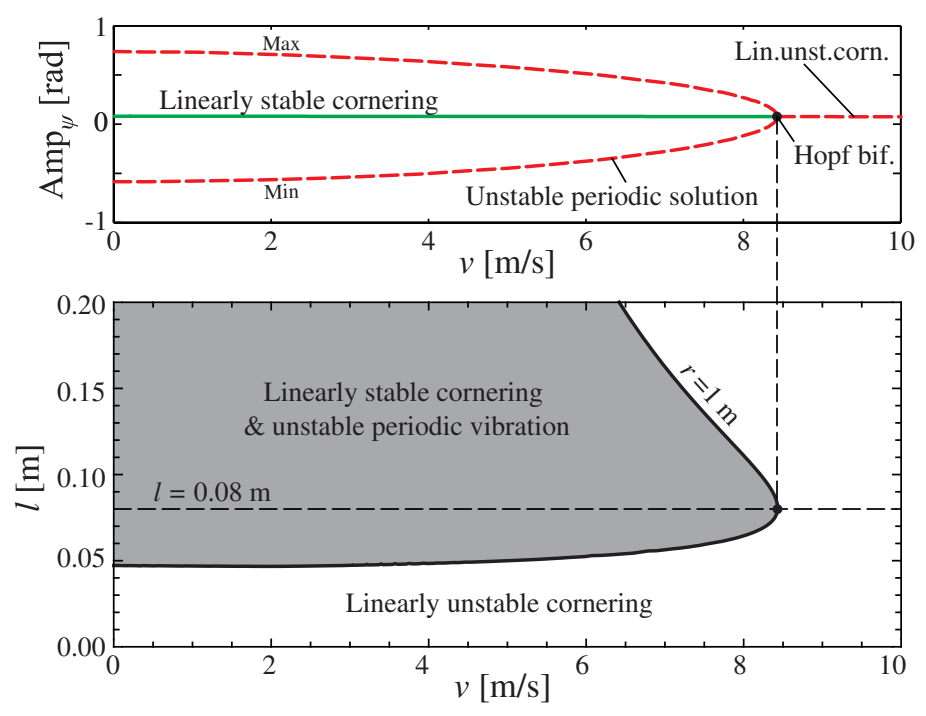

Figure 5. Linear stability chart with a bifurcation diagram

The linear stability boundary of uniform motion corresponds to a Hopf bifurcation, which sense is determined numerically in our study. Figure 5 shows the bifurcation diagram together with the linear stability chart. The towing speed is used as bifurcation parameter and the amplitude of the caster angle $\mathrm{Amp}_{\psi}$ is used to characterize the uniform motion and the periodic solutions. In the bifurcation diagram, stable and unstable solutions are plotted by solid (green) and dashed (red) curves, respectively. As it can be seen, the sense of the Hopf bifurcation is subcritical (i.e. unstable limitcycle exists), which means that large enough perturbation can lead to unwanted vibrations even in the linearly stable domain. The upper and lower bifurcation branches refers to the maximal and minimal caster angles that corresponds to the unstable periodic solution.

Let us investigate the effect of the cornering radius on the unstable periodic solution. Figure 6 shows bifurcation diagrams for the caster length $l=0.08 \mathrm{~m}$. Bifurcation branches are plotted for different cornering radii. In order to make the comparison easier, the angle of the caster is calculated relative to the uniform motion, i.e. $A \operatorname{mp}_{\psi-\psi_{0}}$ is calculated and illustrated in these bifurcation diagrams. Thus, the basins of the attraction of the uniform motion can be compared. As it can be observed in the figure, the cornering radius does not have relevant effect on the amplitude of the unstable periodic solutions at low speed far from the linear stability boundary. A small asymmetry of the periodic solution can also be observed, namely, perturbations that have 

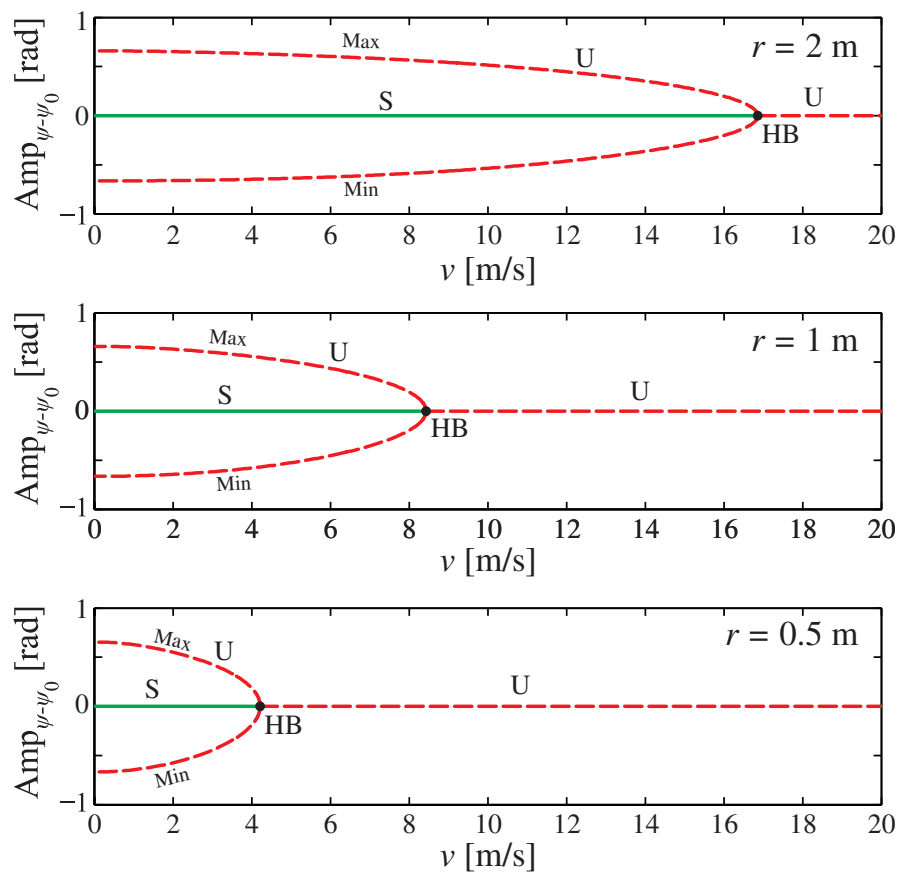

Figure 6. Bifurcation diagrams for different cornering radii

the same magnitude and different directions may lead to qualitatively different motions. But this asymmetry is practically not relevant in the investigated parameter domain.

\section{CONCLUSIONS}

It can be established that the cornering manoeuvre of the towed rigid wheel is a worse scenario than the rectilinear motion at least with respect to linear stability. Former studies also showed that linear stability analyses can not discover all the important dynamical behaviour of towed wheels. Bifurcation analyses (see, for example, Takács et al. (2008)) can unhidden parameter ranges, where the linear stability is guaranteed but large amplitude vibrations coexist too. The non-linear analysis of the cornering manoeuvre does not provide similar properties of the towed wheels without considering any damping or the sliding of the wheel.

Acknowledgement This research was partly supported by the János Bolyai Research Scholarship of the Hungarian Academy of Sciences and by the Hungarian National Science Foundation under grant no. OTKA PD105442.

\section{REFERENCES}

Besselink, I. J. M. (2000). Shimmy of Aircraft Main Landing Gears. Ph. D. thesis, Technical University of Delft, The Netherlands.

Cossalter, V. (2006). Motorcycle Dynamics. Lulu Editor.

Doedel, E. J., A. R. Champneys, T. F. Fairgrieve, Y. A. Kuznetsov, B. Sandstede, \& X. Wang (1997). AUTO97: Continuation and bifurcation software for ordinary differential equations. Technical report, Department of Computer Science, Concordia University. http://indy.cs.concordia.ca/auto/.

Pacejka, H. B. (2002). Tyre and Vehicle Dynamics. Linacre House, Jordan Hill, Oxford OX2 8DP, 200 Wheeler Road, Burlington MA 01803: Elsevier Butterworth-Heinemann.

Sharp, R. S., S. Evangelou, \& D. J. N. Limebeer (2004). Advances in the modelling of motorcycle dynamics. Multibody System Dynamics 12(3), 251-283.

Takács, D., G. Stépán, \& S. J. Hogan (2008, April). Isolated large amplitude periodic motions of towed rigid wheels. Nonlinear Dynamics 52(1-2), 27-34. 
Terkovics, N., S. Neild, M. Lowenberg, \& B. Krauskpof (2012). Bifurcation analysis of a coupled nose landing gear-fuselage system. In Proceedings of AIAA 2012, Minneapolis, Minnesote, USA, pp. 1-14. AIAA. paper No. AIAA 2012-4731. 